\title{
Safety Simulation Analyses for Shot Solid Rocket Motor
}

\author{
Hong-bo Liü, a, Xin-long Chang $^{1}$, Kuan Hu${ }^{1}$, Chun-guo Yue ${ }^{1}$ \\ ${ }^{1}$ Xi'an High-tech Institute, Xi'an, 710025 China; \\ ahuluobu_1979@126.com
}

Keywords: solid rocket motor; solid propellant; shot; numerical simulation

\begin{abstract}
In order to study the safety of a shot SRM, a simulation model was established. Ignition and growth reactive flow equation was applied to describe the ignition process of the impacted propellant. The process of SRM being shot was simulated numerically by use of nonlinear finite element method of fluid dynamics. It was demonstrated that the critical velocity of the shot SRM exploding is $850 \sim 855 \mathrm{~m} / \mathrm{s}$, and the max pressure was in the wave front during the initiation caused by impact.
\end{abstract}

\section{Introduction}

During the moving and launching of wartime missile, its solid rocket motor may be impacted by bullet or shrapnel flying at high speed. The harm of the impact manifest as the mechanical damage to the Motor shell and the damage to the propellant after the bullet penetrating the shell. Therefore, it is essential to analysis and evaluate the safety of a shot SRM by studying its response after being shot.

Popping experiment is the important method to evaluate the safety of the solid propellant under being impacted by bullet or fragments moving at high speed, and a lot of experimental research on the explosives and propellants were carried out at home and abroad. In the process of shooting, high-speed impact from bullet would produce shock waves in the solid propellant. At the same time when the bullet goes through the solid propellant, heat is produced due to friction between them. The combustion or detonation reaction may occur in solid propellant under the action of shock waves and friction heat alone or together. Therefore, the response of the shot engine can be simulated and analyzed in three conditions. to the simulation analysis.

(a) The velocity of the bullet is high enough to cause a immediate detonation inside the engine.

(b) The bullet move at high speed which is not enough to cause a immediate detonation inside the engine, but the propellant is ignited by the heat produced by penetration work and high-speed friction.

(c) The bullet move at low speed which is enough to penetrate the shell but not to cause a reaction, or it don't penetrate the shell.

In the paper, the finite element simulation of the process of SRM being shot was performed by use of LS-DNYA. The process of ignition by shooting was analyzed and the bullet's critical velocity which detonated the SRM was calculated.

\section{Finite element simulation}

\subsection{Ignition and growth model}

For the research on impact initiation process of solid propellant, one of the key issues is to determine the reaction rate function, which make up the behavior and characteristics of the initiation process. 
For the energetic material's response caused by heat transfer problem, Arrhenius law is enough to describe it, which thermolysis rule can more accurately reflect the exothermic decomposition rate. But it don't apply to the description of the response of high-speed mechanical impact and shock waves. Tarver and Lee, based on the theory of hot-spot, through the analysis of test data ,concluded that shock initiation need to be described using at least three stages, which are the nucleation, growth and convergence of the hot-spot. They proposed the Lee-Tarver trinomial ignition and growth model, which trinomial express, in order, ignition, combustion and fast reaction as following:

$$
\frac{d F}{d t}=I(1-F)^{b}\left(\frac{\rho}{\rho_{0}}-1-a\right)^{x}+G_{1}(1-F)^{c} F^{d} P^{y}+G_{2}(1-F)^{e} F^{g} P^{z}
$$

where $F$, t, $\rho, \rho_{0}$ and $P$ are the Explosive reaction degree, time, density, initial density, and pressure, and $I, G_{1}, G_{2}, x, b, c, d, y, e, g$ and $z$ are constant. The terms of the polynomial, in order, reflect the effect of the compression strength on the ignition, effect of the burn of the energetic material on the reaction rate and relatively slow diffusion-controlled reaction after the main reaction.

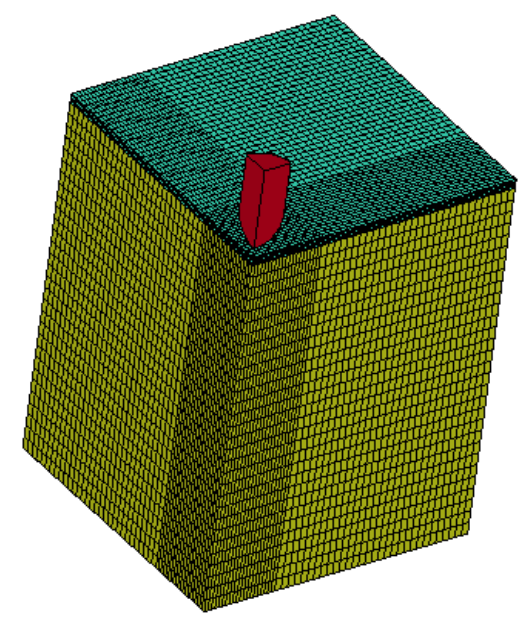

Fig. 1 Bullet impacting the shell and mesh generation of the propellant

\section{2 geometric model}

The mechanism of bullet impacting SRM is similar to that of bullet impacting shelled explosive, except that the shell and heat insulativity (mainly shell) greatly affect the velocity, temperature and shape, which is slightly more complicated. Although congeneric solid propellant, SRM contains more oxidizer grains, is less homogeneous and more insensitive than explosive. To facilitate modeling and calculation in this paper, a steel plate $5 \mathrm{~mm}$ thick was adopted as the motor shell, the thick of grain was set to $20 \mathrm{~cm}$, and the influence of heat-insulating Layer was ignored. Since SRM is much bigger than warhead, to use the plane instead of the curved surface was feasible. Assuming the propellant was homogeneous, its reaction rate was described with Lee-Tarver ignition and growth equation above. A quarter three-dimensional model and its grid map was presented in Fig 1.ANSYS/LS-DYNA program was used to simulate the progress of bullet impacting SRM. JOHNSON_COOK plastic material model with fracture failure was adopted as the shells of bullet and SRM, which relation between pressure and volume could be described with GRUNEISEN equation state as following: 


$$
p=\frac{\rho_{0} C^{2} \mu\left[1+\left(1-\frac{\gamma_{0}}{2}\right) \mu-\frac{a}{2} \mu^{2}\right]}{\left[1-\left(S_{1}-1\right) \mu-S_{2} \frac{\mu^{2}}{\mu+1}-S_{3} \frac{\mu^{3}}{(\mu+1)^{2}}\right]}+\left(\gamma_{0}+a \mu\right) E
$$

where $C$ is the intercept of vs-vp curve, $S_{1}, S_{2}, S_{3}$ are its slope factor, $\gamma_{0}$ is GRUNEISEN constant, $a$ is the first-order volume correction value of $\gamma_{0}$. and $\mu=\frac{\rho}{\rho_{0}}-1$, and $E$ is initial internal energy.

ELASTIC_PLASTIC_HYDRO model was adopted as propellant material, and its reaction rate was described with Lee-Tarver ignition and growth equation, where the parameters were taken from literature [6] as Tab 1.

Table 1 The parameters of reaction rate equation of propellant ignition and growth model

\begin{tabular}{cccccccccccc}
\hline $\mathrm{I}$ & $\mathrm{b}$ & $\mathrm{a}$ & $\mathrm{x}$ & $\mathrm{G}_{1}$ & $\mathrm{c}$ & $\mathrm{d}$ & $\mathrm{y}$ & $\mathrm{G}_{2}$ & $\mathrm{e}$ & $\mathrm{G}_{2}$ & $\mathrm{z}$ \\
\hline $7.4 \mathrm{E} 11$ & 0.67 & 0 & 800 & 7 & 0.67 & 0.111 & 1.5 & 800 & 0.333 & 1.0 & 2.0 \\
\hline
\end{tabular}

\section{Result analysis}

LS-DYNA post-processing software is capable of real time monitoring for the change of all node positions and pressure etc in the course of the impact. Whether the propellant explodes can be estimated by observing the deformation of it as well as its shell and the variation of the pressure in it. Firstly, let the bullet impact the SRM at an initial velocity of $900 \mathrm{~m} / \mathrm{s}$. The screenshot of the 8 moments' nodes change in the course of the impact is in Fig 2, where we can clearly see that serious deformation occurred in the shell and propellant at $100 \mu \mathrm{s}$. Thus it was considered that the SRM may have explored under the impact of this velocity.

By means of further analysis of pressure change and extrema inside propellant in the course of the impact, more accurate judgment can be obtained. Pressure nephogram of shock wave surface inside propellant of various times is in Fig 3, where it could be seen that at $15 \mu$ s the peak pressure of the propellant firstly impacted had been over $1.4 G P a$, and The calculation results from literature [6] indicate that this kind of propellant will be initiated when the pressure of shock wave reaches $3 G P a$. At $40 \mu \mathrm{s}$, the peak pressure was over $10 \mathrm{GPa}$. In view of that the speed of bullet had been much lower at this time obviously, so great pressure could not be caused by impact of bullet but only by detonation of propellant, which determined that propellant has explored. In the whole process of impact and initiation, the peak pressure of propellant appeared in interface between bullet and propellant only at the initial stage, and then all in shock wave surface which shape was very similar to bullet'(Fig 3). Through extracting the peak pressure inside propellant at various times, pressure change and extrema inside propellant in the course of the impact could be grasped concretely. Propellant pressure time curve is shown in Fig 4. 


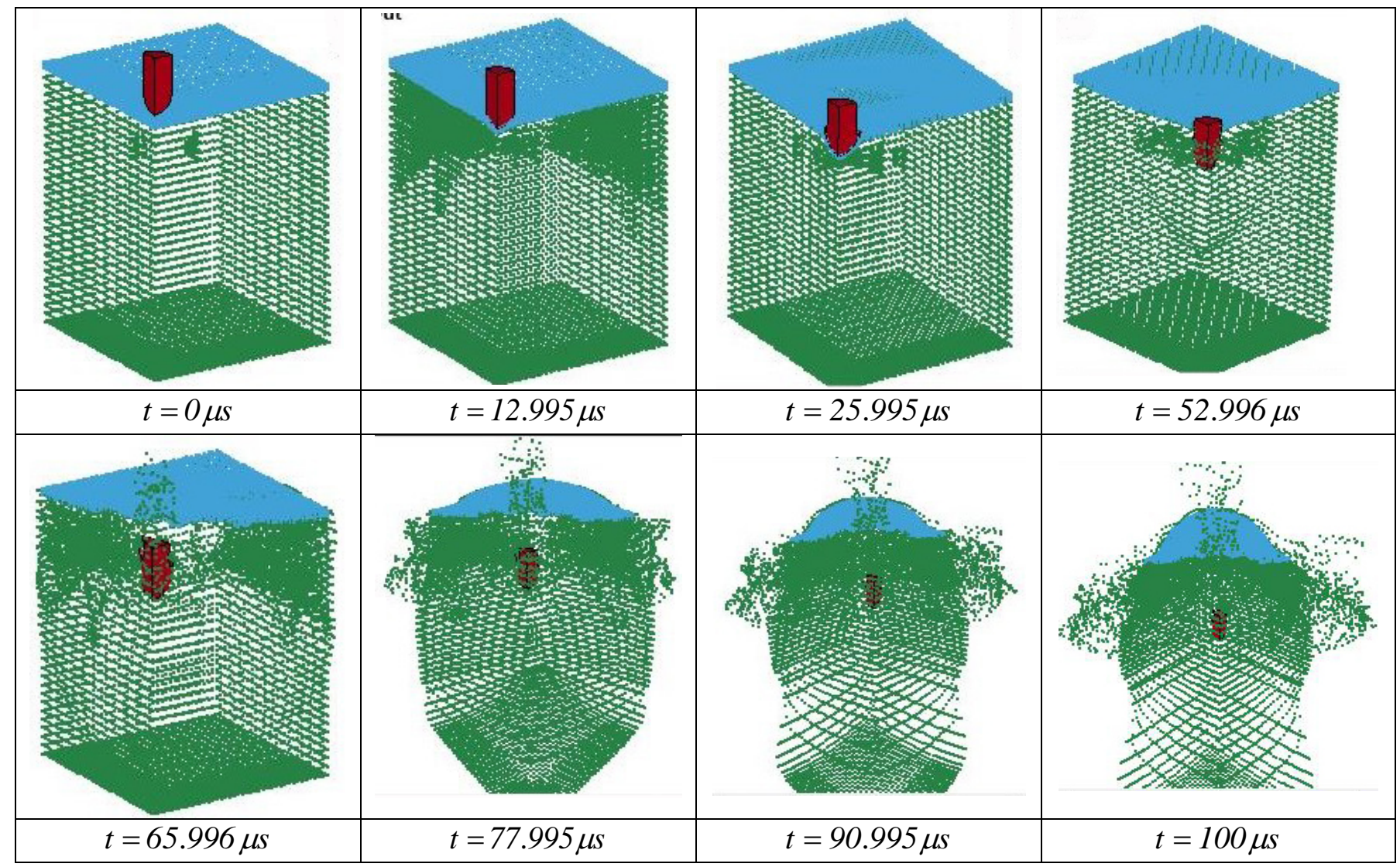

Fig. 2 Deformation of the shell and propellant in the course of the impact

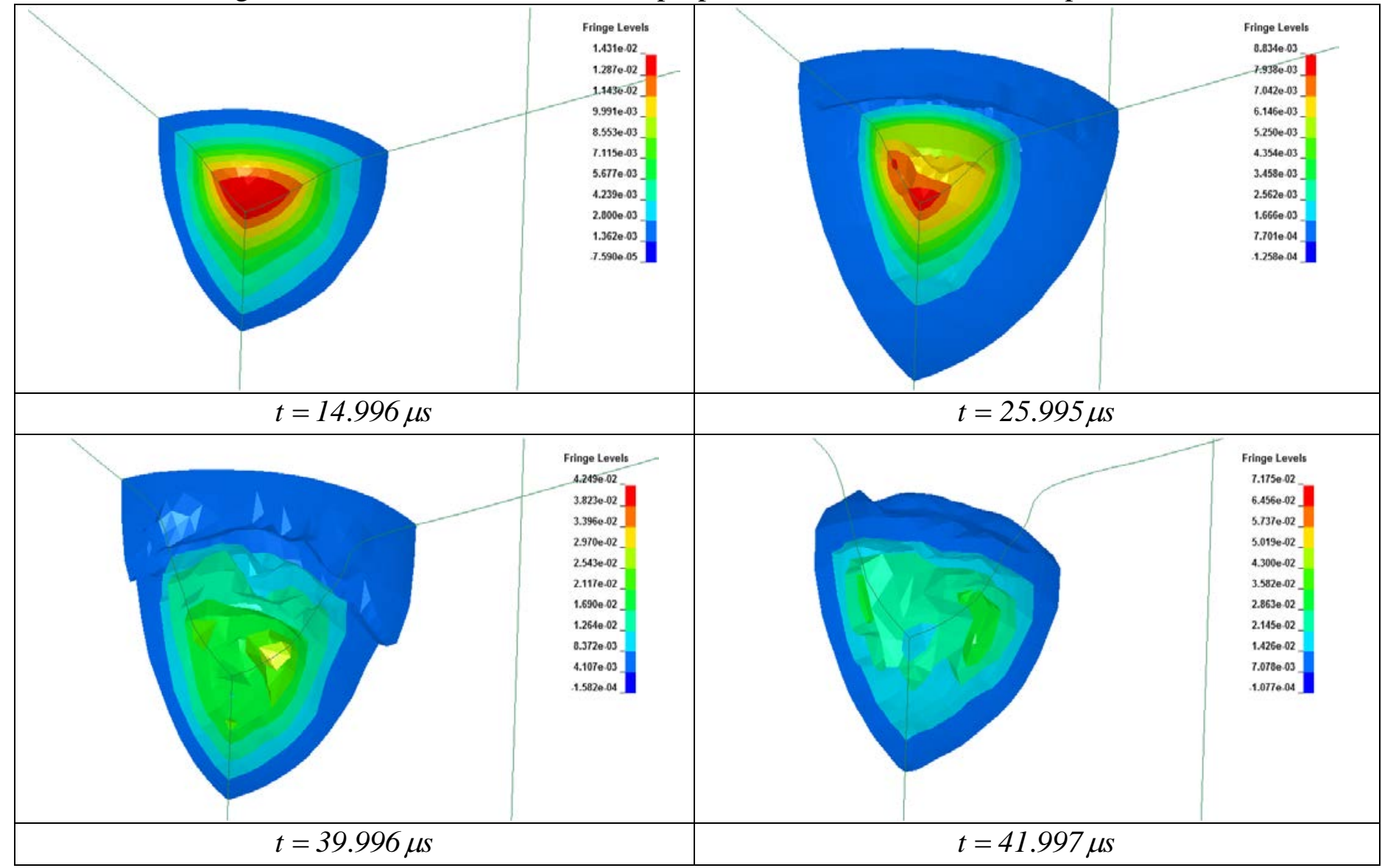




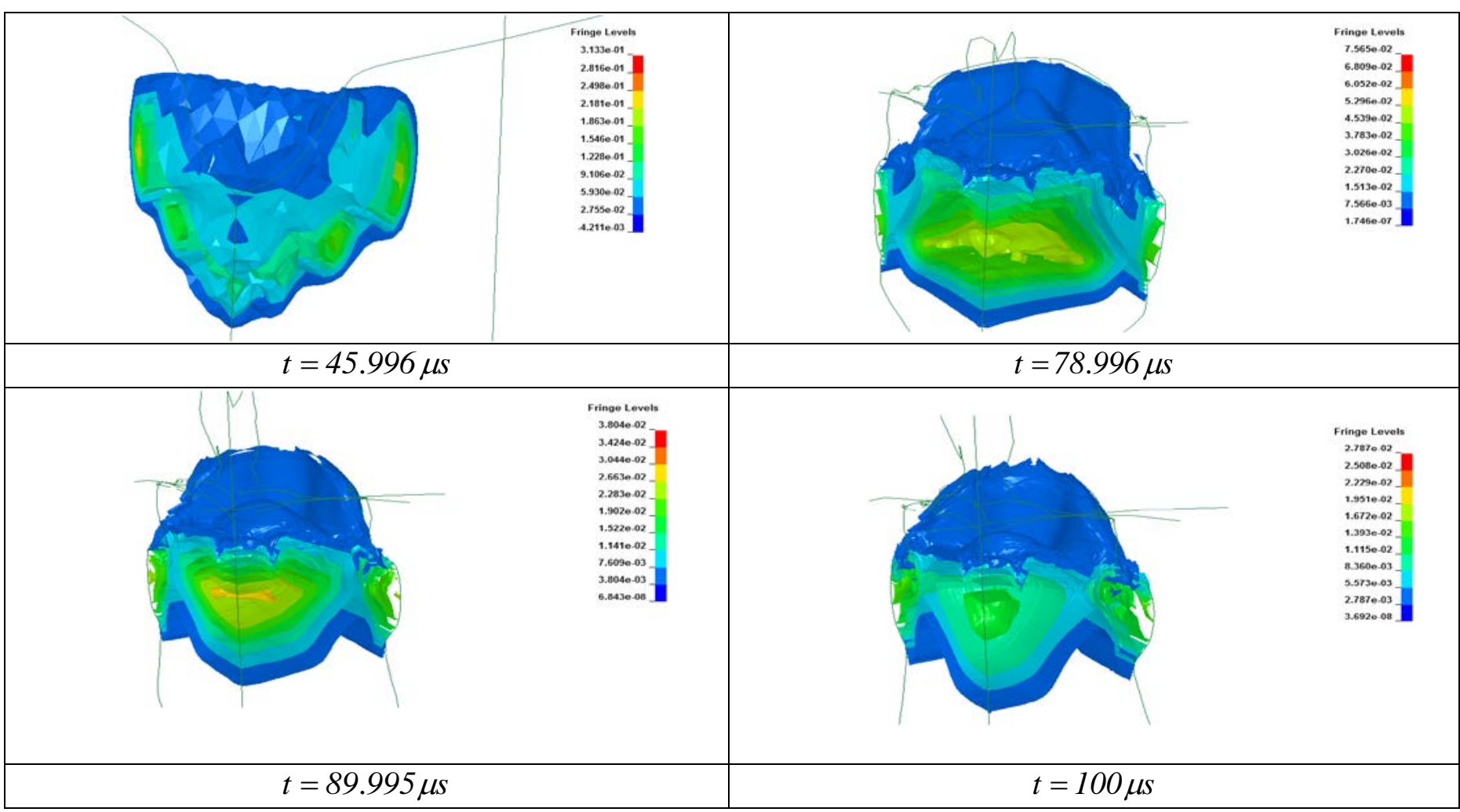

Fig. 3 Nephogram of shock wave surface inside propellant of various times

It can be seen from the curve that an outstanding pressure peak and several peaks appeared during the impact process, and the duration when pressure was over $30 \mathrm{GPa}$ lasted about $40 \mu \mathrm{s}$, which could only be caused by detonation of the whole propellant. It indicates that this impact speed had lead to the detonation of propellant.

After setting the parameters of materials, trial was carried out after modifying the bullet's initial speed in $\mathrm{K}$ file successive by means of rising and falling. Then the bullet's critical velocity $V_{C R}$ which detonated the SRM could be obtained by restart of LS-DYNA and later analysis. In order to obtain the critical velocity, a smaller velocity value should be used to calculate. If this time explosion occur, reduce the value. If not, increase it properly. The process will repeat until the critical velocity is tested out. Fig 5 gives the peak pressure change inside propellant when the bullet's velocity was close to the critical point which detonated the SRM. The three curves of Fig 5 show, in order, the responses of propellant under bullet' impact at various speeds of $870 \mathrm{~m} / \mathrm{s}, 850 \mathrm{~m} / \mathrm{s}$ and $820 \mathrm{~m} / \mathrm{s}$. 


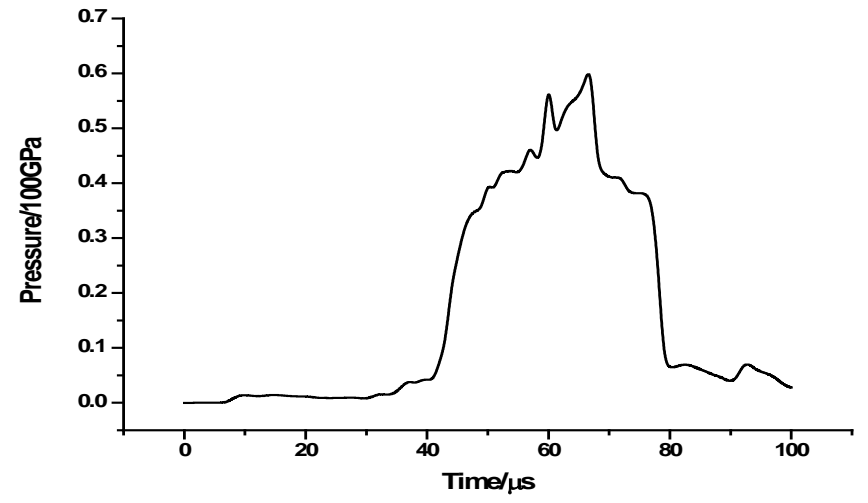

Fig. 4 Propellant maximum pressure time curve in the course of the impact

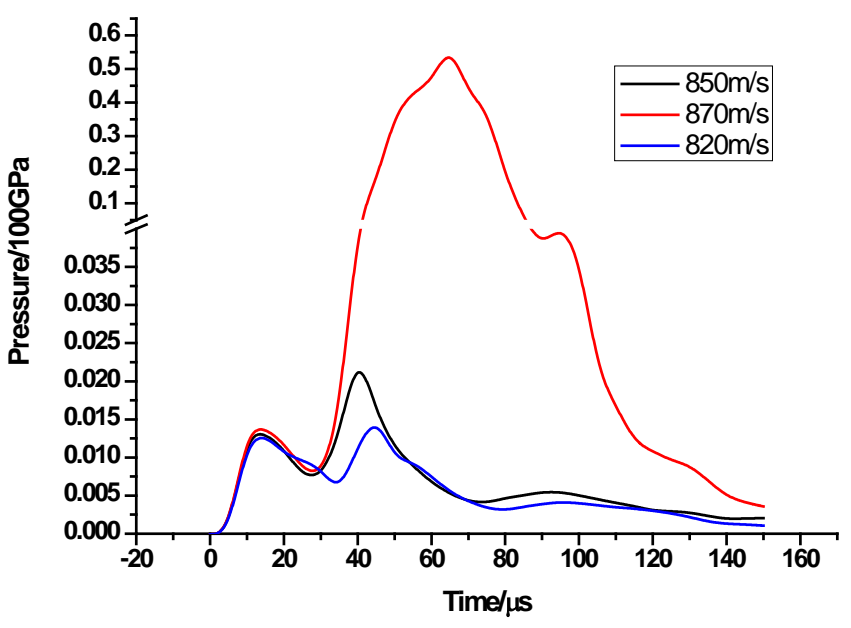

Fig.5 Propellant maximum pressure time curve of various impact velocities

Any of the curve in Fig 5 has two obvious peak waves which corresponding time can be analyzed simply to know that first peak wave was formed when bullet impacting shell and second when bullet penetrating shell. The peak pressure inside propellant was not over $3 G P a$ and fall quickly when the velocity of bullet was $850 \mathrm{~m} / \mathrm{s}$ or $820 \mathrm{~m} / \mathrm{s}$. But the pressure rise sharply to dozens of $G P a$ and lasted for a long time when the velocity was $870 \mathrm{~m} / \mathrm{s}$.

Trial results of various velocities is shown in Tab. 2, where we can see that bullet's critical velocity $V_{C R}$ which detonates the $S R M$ was in the range of $850 \mathrm{~m} / \mathrm{s}$ and $855 \mathrm{~m} / \mathrm{s}$.

Table 2 Trial results of $V_{C R}$ of various impact velocities

\begin{tabular}{cccc}
\hline $\begin{array}{c}\text { Bullet velocity } \\
(m / s)\end{array}$ & Propellant exploded & $\begin{array}{c}\text { Impact time } \\
(\mu \mathrm{s})\end{array}$ & $\begin{array}{c}\text { Propellant maximum pressure } \\
\left(G P_{a}\right)\end{array}$ \\
\hline 1000 & YES & 120 & 58.32 \\
700 & NO & 400 & 1.76 \\
900 & YES & 100 & 57.20 \\
800 & NO & 300 & 2.087 \\
850 & NO & 200 & 2.446 \\
870 & YES & 250 & 54.49 \\
860 & YES & 250 & 54.16 \\
855 & YES & 250 & 54.22 \\
\hline
\end{tabular}

\section{Summary}

In the paper, the finite element model of SRM being shot was established by use of LS-DNYA, and the process of ignition by shooting was simulated. Through these studies, following conclusions were obtained.

(a) Bullet's critical velocity which impacts and detonates the SRM was in the range of $850 \mathrm{~m} / \mathrm{s}$ and $855 \mathrm{~m} / \mathrm{s}$.

(b) In the process of impact and initiation, the peak pressure of propellant appears in shock wave surface which shape was very similar to bullet'. 


\section{References}

[1] Zhuang. J. H, Mao. J, Zhang. W. H, etc, Numerical simulation of the gunshot process of solid rocket motor, J. Journal of Solid Rocket Technology. 2009,32(4):422-426.

[2] Shen. W, Wang. W, Zhang. J, etc, Safety evaluation for long stored SRM, J. Journal of Aerospace Power. 2013,28(4):930-934.

[3] Chen. G. N, Investigation of the safety for Solid Rocket Motor Under Mechanical Impact, D. Doctoral dissertation of graduate school of National University of Defense Technology. 2005.

[4] Lee. EL, Tarver. CM, Phenomenological model of shock initiation in heterogeneous explosives, J. Physics of Fluids.1980,23(12):2362.

[5] Wu. J. Y, Ch. L, Lu. J. Y, etc, Research on Shock Initiation of the High Energy Solid Propellants, J. Acta Armamentarii. 2008,29(11):1315-1319. 